\title{
Valley selective tunneling transistor based on valley discontinuities in AGaAs heterostructures
}

\author{
Jasprit Singh \\ Center for High Frequency Microelectronics, Department of Electrical Engineering and Computer Science, \\ The University of Michigan, An Arbor, Michigan 48109
}

(Received 13 April 1989; accepted for publication 12 October 1989)

\begin{abstract}
The conduction-band discontinuities in $\mathrm{Al}_{x} \mathrm{Ga}_{1}, \mathrm{As}_{x} / \mathrm{Al}_{y} \mathrm{Ga}_{1}, \mathrm{As}_{\mathrm{s}}$ heterostructure for various valleys $(\Gamma, L, X)$ are utilized to conceive of a valley selective tunneling transistor. At low temperature, the perpendicular tunneling current is very small because of the large $\Gamma-\Gamma$ discontinuity and the small prefactor for $\Gamma$ to $X$ tunneling. However, if the valley population is altered by applying a lateral bias to the emitter, one can dramatically alter the perpendicular current since the $X-X$ discontinuity is nonexistent and the $L-L$ discontinuity is very small. By choosing proper values of $x$ and $y$ in the heterostructure, a very large current (power) change can be produced in the ON and OFF states.
\end{abstract}

Among other things, an important outcome of recent studies on tunneling in heterostructures has been the demonstration that at low temperatures ( $77 \mathrm{~K})$, the tunneling current is controlled by the "same valley" barrier. 1 "Thus the electron tunneling process from GaAs across an AlAs barrier is controlled by the $\Gamma_{\text {GaAs }}-\Gamma_{\text {AlAs }}$ conduction-band-edge bamier even though the $\Gamma_{\text {GaAs }}-X_{\text {Alas }}$ barrier is very small. ${ }^{3,4}$ Although the detailed understanding on this sibject is still emerging, it is known that this related to the momentur: change required in crossing from $\Gamma-X$ (any dissimiar) valley. The $\Gamma \cdot X$ transition is not forbidcen, but at $77 \mathrm{~K}$, it is expected to have a $10^{-4}$ imes smaller prefactor than the valley conserving tunneling current. ${ }^{3,5}$ th has also been established that the GaAs-AIAs system has a rather unusual band Sineup in the conduction band, with the conduction-band discontinuity ied to the $\Gamma_{\text {GaAs }}-\Gamma_{\text {AlAs }}$ discontinuity and not to the band-gap diferences. ${ }^{6}-8$ The valley lineups and conduction-bard discontinuities are sketched in Fig. 1 for the three lowest conduction-band valleys in the AlGaAs system. Because of the commutative relation satisfed by the discontinuities, Fig. 1 can be used to calculate the discontinuity for any heterostructure composition. The unusual lineup alows us to make some simple observations on the $\mathrm{Al}_{x} \mathrm{Ga}_{1-x} \mathrm{As} / \mathrm{Al}_{y} \mathrm{Ga}_{1-y}$ As system with $x<y$ : (i) while the $\Gamma-\Gamma$ discontinuity increases with increasing $y-x$, the $X-X$ discontinuity decreases. The $L-L$ discontinuiry also increases, but at a much smaller rate. Thus, with proper choice of $x$ and $y$, one can have a large $\zeta-\Gamma$ separation and a very small $L-L$ separation and $n o X-X$ separation. We will focus on the heterostructures where $x \leqslant 0.4$ (i.e., direct) and $y \geqslant 0.6$ (indirect).

At equilibrium when most of the carriers (for $x<0.4$ ) are in the $\Gamma$ valley in $\mathrm{Al}_{x} \mathrm{Ga}_{1}{ }_{x} \mathrm{As}$, the inelastic tunneling to the higher Al side is minimal even in the presence of a large transverse field as shown in Fig. 2(a). If somehow, now the carriers conld be transferred to the higher $X$ or $L$ valleys in $\mathrm{Al} \mathrm{Ga}_{1-x} \mathrm{As}$, a significant portion of these carriers could move out into the higher $\mathrm{Al}$ content side. This change in valley occupation is reasonably straightforward to do by simply subjecting the carriers in $\mathrm{A} 1_{x} \mathrm{Ga}_{1-x} \mathrm{As}$ to a bias (the control or signal bias) in the lateral direction as shown in Fig. 2(b). Theoretical calculations using Monte Carlo tech * niques show that the electron temperature rises with increased bias changing the population of upper valleys.

This letter utilizes the above concepts to design a transistor in which the (i) signal or control voltage changes the valley population; (ii) the peculiar conduction-band lineup of the AlGaAs system allows for a large $\Gamma-\Gamma$ but a small or negative $L-L$ and $X-X$ discontinuity, respectively, across a heterostructure; and (iii) the valley selective nature of tunneling then allows for a large change in the perpendicular current in response to the signal voltage. Figure 3 shows a schematic of the device conceived. It may be noted that the various components on which this device is based have been observed in diferent forms already. Here we merely exploit these efiects to generate a possibly useful device. On first look, our device concept may appear similar to the read space-charge transfer concept, ${ }^{10}$ but we note that we use the unusual valley lineups and momentum selection rules to obtain a highly noninear response.

The relevant equations for the conduction-band disconfiruities for the $\mathrm{Al}_{x} \mathrm{Ga}_{1}{ }_{-x} \mathrm{As}$ system are (assuming $\sim 65 \%$ of the $\mathbb{T}$ valley band-gap change drops in the conduction band)

$$
\begin{aligned}
& \Delta E_{c}^{\Gamma-\Gamma}(x, y) \cong 0.9(y-x), \\
& \Delta E_{c}^{X-X}(x, y) \cong-0.3(y-x), \\
& \Delta E_{c}^{L-L}(x, y) \cong 0.2(y-x) .
\end{aligned}
$$

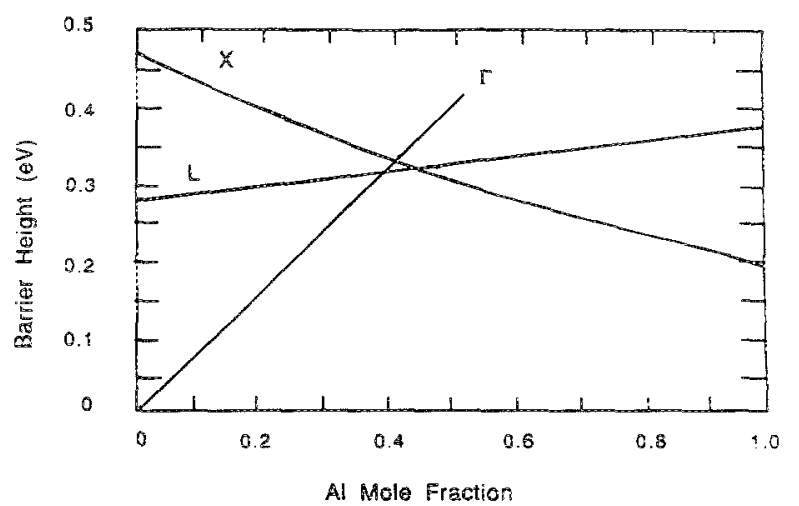

FIG. 1. Variation of the band-edge discontinuties for diferent valleys in AlGaAs-based heterostructures. 


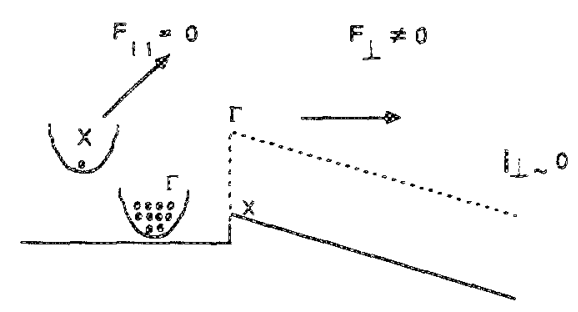

(a)

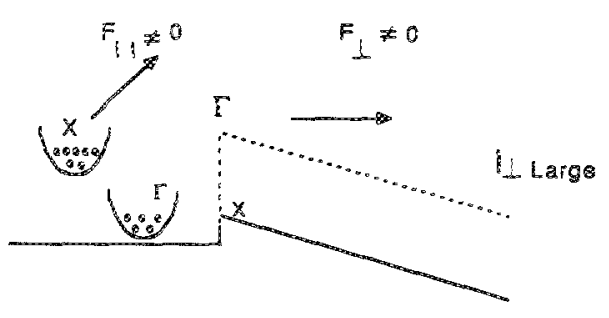

(b)

FGG. 2. (a) Schematic diagram showing how at zero lateral field, the perpendicular current $I_{\text {in }}$ is negligible in spite of the strong transverse elcctric fleld; (b) A schematic showing how a finite lateral field allows carriers to move to the upper valley and contribute to $I_{1}$.

Note that while the $\Gamma-\Gamma$ and $L-L$ discontinuities increase with increasing Al composition, the $X-X$ discontinuity decreases. A number of interesing configurations then present themselves for utilizing these special lineup properties.

The total iransmission rate for an electron to tunnel across the barrier is then

$$
\begin{aligned}
& P \cong \alpha_{\Gamma}\left(F_{11}\right)\left[A_{1} \exp \left[\frac{-4}{3}\left(\frac{2 m_{\mathrm{T}}}{n^{2}}\right)^{1 / 2} \frac{\left[\Delta E^{\Gamma-r}(x, y)\right]^{3 / 2}}{e F_{\perp}}\right]\right. \\
& +B_{1} \exp \left[\frac{-4}{3}\left(\frac{2 m_{X}}{n_{2}}\right)^{1 / 2}\left[\frac{\left[\Delta E^{\Gamma-X}(x, y)\right]^{3 / 2}}{e E}\right]\right. \\
& +c_{L}\left(F_{11}\right)\left\{A_{2} \exp \left[\frac{-4}{3}\left(\frac{2 m_{L}}{h^{2}}\right)^{1 / 2} \frac{\left[\Delta E^{1-L}(x, y)\right]^{3 / 2}}{e F_{1}}\right]\right\} \\
& +\alpha_{X}\left(F_{11}\right) A_{3} .
\end{aligned}
$$

Here $F_{1}$ is the transverse electric field, $\alpha_{i}\left(F_{11}\right)$ is the occupation of the th valley as a function of the lateral field $F_{1:} ; A^{\prime} \mathrm{s}$ are the attemp frequencies for the similar valleys and $B_{1}$ is the attempt frequency for the dissimilar $\Gamma \rightarrow X$ tumening $\left(B_{1} \sim 10^{-4} A_{1}\right)$. We have ignored $\Gamma \rightarrow L, L \rightarrow \Gamma$, and $X \rightarrow \Gamma$ tunneling processes due to their much smaller contributions. We also note that the $\triangle E$ discontinuities are measured with respect to the initial transverse energies of the electron. The current can be calculated as for the current across a junction" except that the $a$ 's are to be calculated by Monte Carlo methods.

Let us consider a structure $n-\mathrm{Al}_{0.33} \mathrm{Ga}_{0.57} \mathrm{As} /$ $i-\mathrm{A} 1_{0.6} \mathrm{G} \Omega_{0.4}$ As. The $\Gamma-X$ separation in the $n$ emitter is only $\sim 0.09 \mathrm{eV}$ (in contrast to $\sim 0.45 \mathrm{eV}$ in GaAs) so that carriers cam be pushed to the $X$ valley by a small $V_{s}$. The various parameters used are: $\Gamma-\Gamma$ discontinuity $=0.25 \mathrm{eV}, T-X$ discontinuicy $=0.05 \mathrm{eV}, L-L$ discontinuity $=0.03 \mathrm{eV}, X-X$ discontinuity $=0.03-0.075 \mathrm{eV}$, and $V_{T}=5 \mathrm{~V}$ (across a $0.5 \% \mathrm{~m}$ iregion).

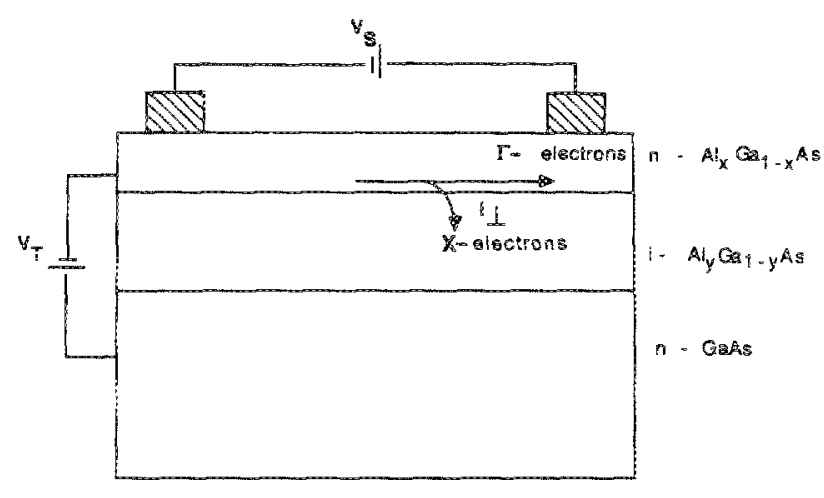

FIG. 3. Schematic of the device structure studied to calculate the ON-OFF: properties.

At $77 \mathrm{~K}$, the dominant path for the transverse current wher the lateral bias $V_{s}$ is zero is via the dissimilar valley tunneling, $\Gamma \rightarrow X$. However, as the lateral bias is increased, the $X$ valley occupation increases. Although the field is lateral, $X$ valley carriers in the emitter diffuse to the interface where thoy are collected in the collector due to the high transwerse bias and negative $X-X$ discontinuity. The $L \rightarrow L$ current is also quite high at nonzero $V_{s}$ values.

if we assume that the contact spacing on the emitter is $1.0 \mu \mathrm{m}$ we find the following resuits for the transverse current:

$$
\begin{aligned}
& \text { at } 77 \mathrm{~K}: I_{1}\left(V_{s}=0\right): I_{1}\left(V_{s}=1.0 \mathrm{~V}\right) \cong 1: 10^{5} \\
& \text { at } 300 \mathrm{~K}: I_{1}\left(V_{s}=0\right): I_{1}\left(V_{s}=1.0 \mathrm{~V}\right) \cong 1: 10
\end{aligned}
$$

The relative change is small at rom temperature becalse of the high thermal occupation value for $X$ and $L$ electrons even at $V_{s}=0$.

An extremely nonlinear change occurs as one goes from the ofF $\left(V_{s}=0\right)$ to the oN state. In a real structure, the change may not be as dramatic due to defect-assisted tunneling, etc., but nevertheless, a large change should be observed. Since the $\Gamma-Z$ separation can easiy be altered, one can optimize devices for operation temperature or operating bias $V_{s}$. Studies of these kinds will be reported elsewhere.

In summary, we have discussed a simple device structure which utilizes the peculiar band lineup for the various valleys in the AlGaAs system. The device would be interesting as a transistor as well as a structure to throw dight on how carrier tunneling occurs when the electron distribution function is skewed perpendicular to the barrier.

This work was supported by the U.S. Amy Research Offee URI Program DAAL03-87-R-0007. Discussions with Sandip Tiwari and George Haddad are gratefully acknowledged.

T. E. Mendez, W. 1. Wang, E. Calleja, and C. E. T. Gonzalvex dá Silva, Appl. Plyys. Lete. 50, 1263 (1987).

2M. Tsuchya and Tr. Sakaki, Appl. Phys. Lett. 50, 1503 (1987).

${ }^{3}$ E. E. Mendez, E. Calleja, and W. I. Wang, Appi. Fhys. Lett. 53, 977 (1988).

"W. D. Goodhue, T. C. Solner, H. Q. Le, E. R. Brown, and B. A. Vojak, Appl. 1pys. Lett .49, 1086 (1986).

5P. J. Price, Surf. Sci. 196,394 ( 1988$)$. 
'D. Arndal, A. Ketterson, T. Henderson, J. Khem, and H. Morkos, Appl. Phys. Leti. 45,1237 (1984).

7. Batey, S. L. Wright, and D. J. DiMaria, J. Appl. Phys. 57, 484 (1985).

${ }^{8}$ T. J. Drummond and I. J. Fritz, Appl. Phys. Lett. 47, 284 (1985).

W. Fawcett, A. D. Boaroman, and S. Swain, J. Phys. Chem. Solids 31,
$1963(1970)$

"K. Hess, H. Morkoc, H. Shichijo, and B. G. Strectman, Appl. Phys. Lett. $35,469(1979)$.

'S. M. Sze, Physics of Semicondurtor Devices (Wiley, New York, 1981), p. 254. 